Supplement of Nat. Hazards Earth Syst. Sci., 19, 2565-2582, 2019

https://doi.org/10.5194/nhess-19-2565-2019-supplement

(c) Author(s) 2019. This work is distributed under

the Creative Commons Attribution 4.0 License.

(c) (1)

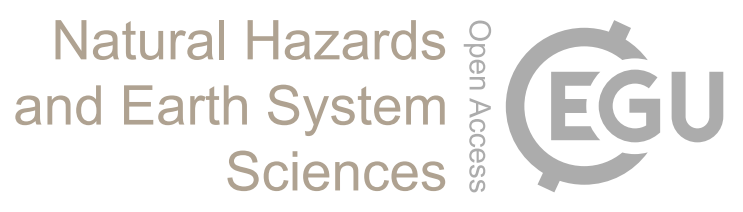

Supplement of

\title{
AGRIDE-c, a conceptual model for the estimation of flood damage to crops: development and implementation
}

Daniela Molinari et al.

Correspondence to: Daniela Molinari (daniela.molinari@polimi.it)

The copyright of individual parts of the supplement might differ from the CC BY 4.0 License. 


\section{Structure of the Supplement}

The Supplement includes, for each principal crops in the Po Plain:

- average yield and price in the Province of Lodi over the last five years;

- distribution of production costs over the year in the case that no flood occurs (i.e. Scenario 0);

- implemented physical model;

- yield reduction and change in production costs on the basis of damage alleviation strategies;

- relative damage for the different combinations of times of flood occurrence (i.e. month), flood intensities (i.e. water depth and flood duration) and damage alleviation strategies

in case of conventional and minimum tillage, when both techniques are possible. Concerning maize crops, only data related to conventional tillage are reported, being those related to minimum tillage included in the main manuscript. 


\section{Maize crops}

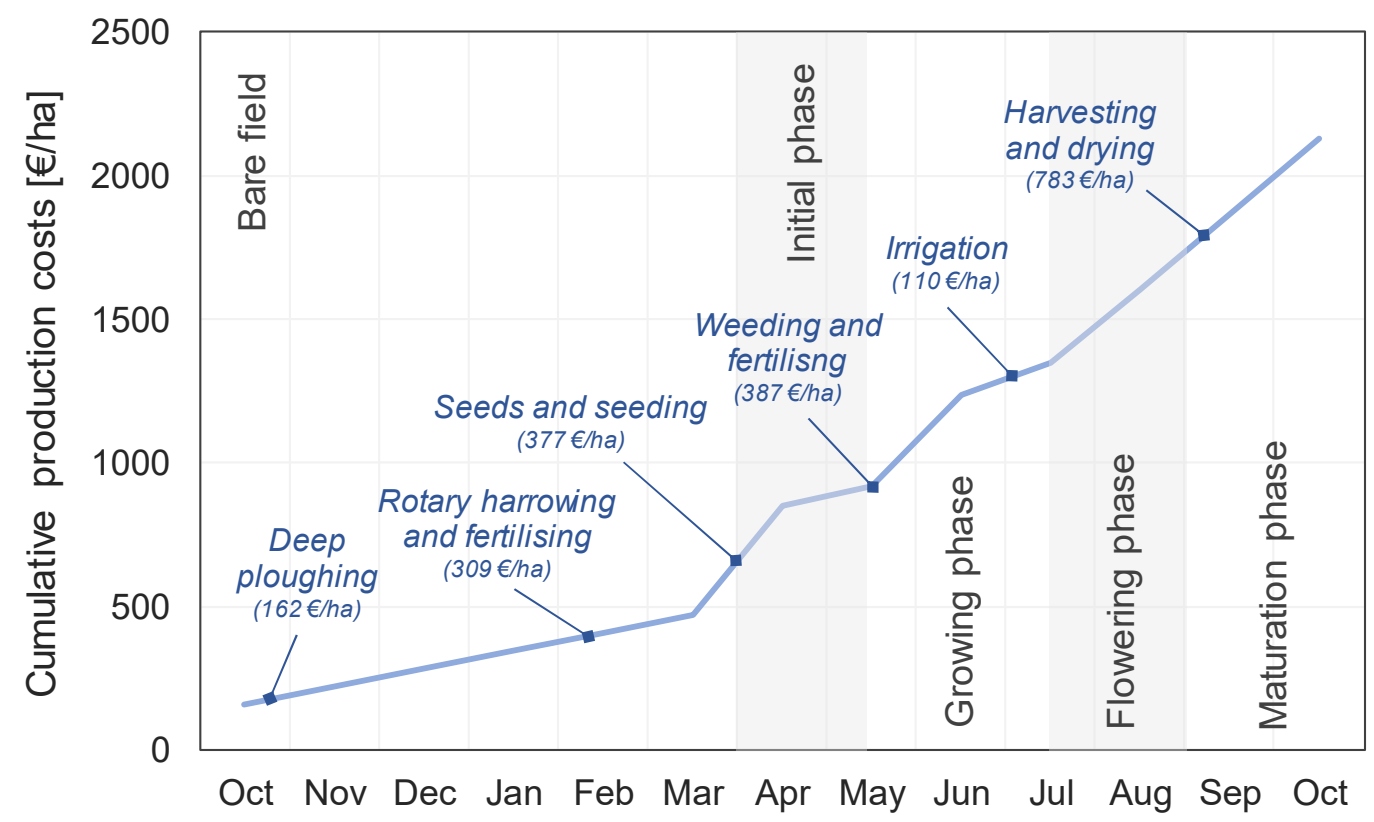

Figure S.1. Distribution of production costs over the year in the Scenario 0 for maize crops, in the case of conventional tillage

Table S.1. Yield reduction and change in production costs on the basis of damage alleviation strategies for maize crops, in case of conventional tillage

\begin{tabular}{|c|c|c|c|c|c|c|c|}
\hline $\begin{array}{l}\text { Time of the } \\
\text { flood }\end{array}$ & $\begin{array}{l}\text { Vegetative } \\
\text { stage }\end{array}$ & $\begin{array}{l}\text { Alleviation } \\
\text { strategy }\end{array}$ & $\begin{array}{c}\text { Yield } \\
\text { reduction } \\
{[\%]}\end{array}$ & Additional costs & $€ /$ ha & Avoided costs & €/ha \\
\hline \multirow{2}{*}{$\begin{array}{l}\text { November - } \\
\text { March }\end{array}$} & \multirow[t]{2}{*}{ Bare field } & \multirow[t]{2}{*}{ Continuation } & \multirow[t]{2}{*}{ I. } & $\begin{array}{l}\text { Soil restoring (sediment } \\
\text { removal and terrain levelling) }\end{array}$ & 500 & & \\
\hline & & & & Deep ploughing & 162 & & \\
\hline \multirow{6}{*}{ April - May } & \multirow{6}{*}{ Initial phase } & \multirow{3}{*}{ Abandoning } & \multirow{3}{*}{100} & \multirow{3}{*}{$\begin{array}{l}\text { Soil restoring (sediment } \\
\text { removal and terrain levelling) }\end{array}$} & \multirow{3}{*}{500} & Weeding and fertilising & 387 \\
\hline & & & & & & Irrigation & 110 \\
\hline & & & & & & Harvesting and drying & 783 \\
\hline & & \multirow{3}{*}{ Reseeding } & \multirow{3}{*}{0} & $\begin{array}{l}\text { Soil restoring (sediment } \\
\text { removal and terrain levelling) }\end{array}$ & 500 & & \\
\hline & & & & $\begin{array}{l}\text { Rotary harrowing and } \\
\text { fertilising }\end{array}$ & 309 & & \\
\hline & & & & Seeds and reseeding & 377 & & \\
\hline \multirow{6}{*}{ June } & \multirow{6}{*}{$\begin{array}{l}\text { Growing } \\
\text { phase }\end{array}$} & Continuation & $\begin{array}{l}\text { see Fig. } 4 \\
\text { of the paper }\end{array}$ & $\begin{array}{l}\text { Soil restoring (sediment } \\
\text { removal and terrain levelling) }\end{array}$ & 500 & & \\
\hline & & \multirow{2}{*}{ Abandoning } & \multirow{2}{*}{100} & \multirow{2}{*}{$\begin{array}{l}\text { Soil restoring (sediment } \\
\text { removal and terrain levelling) }\end{array}$} & \multirow{2}{*}{500} & Irrigation & 110 \\
\hline & & & & & & Harvesting and drying & 783 \\
\hline & & \multirow{3}{*}{ Reseeding } & \multirow{3}{*}{0} & $\begin{array}{l}\text { Soil restoring (sediment } \\
\text { removal and terrain levelling) }\end{array}$ & 500 & & \\
\hline & & & & $\begin{array}{l}\text { Rotary harrowing and } \\
\text { fertilising }\end{array}$ & 309 & & \\
\hline & & & & Seeds and reseeding & 377 & & \\
\hline \multirow{3}{*}{ July - August } & \multirow{3}{*}{$\begin{array}{l}\text { Flowering } \\
\text { phase }\end{array}$} & Continuation & $\begin{array}{l}\text { see Fig. } 4 \\
\text { of the paper }\end{array}$ & $\begin{array}{l}\text { Soil restoring (sediment } \\
\text { removal and terrain levelling) }\end{array}$ & 500 & & \\
\hline & & \multirow{2}{*}{ Abandoning } & \multirow{2}{*}{100} & \multirow{2}{*}{$\begin{array}{l}\text { Soil restoring (sediment } \\
\text { removal and terrain levelling) }\end{array}$} & \multirow{2}{*}{500} & Irrigation & 55 \\
\hline & & & & & & Harvesting and drying & 783 \\
\hline \multirow{2}{*}{$\begin{array}{l}\text { September - } \\
\text { October }\end{array}$} & \multirow{2}{*}{$\begin{array}{l}\text { Maturation } \\
\text { phase }\end{array}$} & Continuation & $\begin{array}{l}\text { see Fig. } 4 \\
\text { of the paper }\end{array}$ & $\begin{array}{l}\text { Soil restoring (sediment } \\
\text { removal and terrain levelling) }\end{array}$ & 500 & & \\
\hline & & Abandoning & 100 & $\begin{array}{l}\text { Soil restoring (sediment } \\
\text { removal and terrain levelling) }\end{array}$ & 500 & Harvesting and drying & 783 \\
\hline
\end{tabular}




\begin{tabular}{|c|c|c|c|c|c|c|c|c|c|c|c|}
\hline \multirow{2}{*}{\multicolumn{2}{|c|}{$\begin{array}{c}\text { Water depth } \\
<130 \mathrm{~cm}\end{array}$}} & \multirow{3}{*}{$\begin{array}{c}\text { Strategy } \\
\text { C }\end{array}$} & \multicolumn{9}{|c|}{ Flood duration [days] } \\
\hline & & & $<5$ & 5 & 6 & 7 & 8 & 9 & 10 & 11 & $>11$ \\
\hline \multirow{9}{*}{ 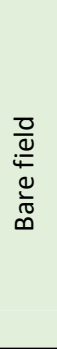 } & \multirow{3}{*}{ Jan } & & \multicolumn{9}{|c|}{$60 \%$} \\
\hline & & $r$ & \multicolumn{9}{|c|}{ - } \\
\hline & & a & \multicolumn{9}{|c|}{-} \\
\hline & \multirow{3}{*}{ Feb } & c & \multicolumn{9}{|c|}{$60 \%$} \\
\hline & & $r$ & \multicolumn{9}{|c|}{-} \\
\hline & & a & \multicolumn{9}{|c|}{-} \\
\hline & \multirow{3}{*}{ Mar } & c & \multicolumn{9}{|c|}{$60 \%$} \\
\hline & & $r$ & \multicolumn{9}{|c|}{-} \\
\hline & & a & \multicolumn{9}{|c|}{-} \\
\hline \multirow{6}{*}{ 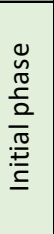 } & \multirow{3}{*}{ Apr } & c & \multicolumn{9}{|c|}{-} \\
\hline & & r & \multicolumn{9}{|c|}{$129 \%$} \\
\hline & & a & \multicolumn{9}{|c|}{$261 \%$} \\
\hline & \multirow{3}{*}{ May } & c & \multicolumn{9}{|c|}{-} \\
\hline & & $r$ & \multicolumn{9}{|c|}{$129 \%$} \\
\hline & & a & & & & & $261 \%$ & & & & \\
\hline$\stackrel{00}{\subseteq}$ & & c & $60 \%$ & $104 \%$ & $149 \%$ & $193 \%$ & $237 \%$ & $282 \%$ & $326 \%$ & $371 \%$ & - \\
\hline 'sa & Jun & $r$ & & & & & $129 \%$ & & & & \\
\hline ப் & & a & - & & & & & & & & \\
\hline & & c & $60 \%$ & $149 \%$ & $237 \%$ & $326 \%$ & & & - & & \\
\hline ఎ & Jul & $r$ & & & & & - & & & & \\
\hline 产 & & a & - & & & & & & & & \\
\hline 茨 & & c & $60 \%$ & $149 \%$ & $237 \%$ & $326 \%$ & & & - & & \\
\hline & Aug & $r$ & & & & & - & & & & \\
\hline & & a & - & & & & & & & & \\
\hline & & c & & & & & $60 \%$ & & & & \\
\hline ๘ & Sep & $r$ & & & & & - & & & & \\
\hline 帝 & & a & & & & & - & & & & \\
\hline$\underset{⿱ 一}{\bar{N}}$ & & c & & & & & $60 \%$ & & & & \\
\hline & Oct & $r$ & & & & & - & & & & \\
\hline & & a & & & & & - & & & & \\
\hline & & c & & & & & $60 \%$ & & & & \\
\hline & Nov & r & & & & & - & & & & \\
\hline 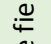 & & a & & & & & - & & & & \\
\hline$\frac{0}{\pi}$ & & c & & & & & $60 \%$ & & & & \\
\hline & Dec & $r$ & & & & & - & & & & \\
\hline & & a & & & & & - & & & & \\
\hline
\end{tabular}

\begin{tabular}{|c|c|c|c|c|c|c|c|c|c|c|c|}
\hline \multirow{2}{*}{\multicolumn{2}{|c|}{\begin{tabular}{|c|} 
Water depth \\
$\geq 130 \mathrm{~cm}$ \\
\end{tabular}}} & \multirow{3}{*}{$\begin{array}{c}\text { Strategy } \\
\mathrm{C}\end{array}$} & \multicolumn{9}{|c|}{ Flood duration [days] } \\
\hline & & & $<5$ & 5 & 6 & 7 & 8 & 9 & 10 & 11 & $>11$ \\
\hline \multirow{9}{*}{ 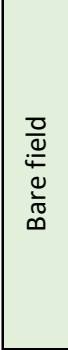 } & \multirow{3}{*}{ Jan } & & \multicolumn{9}{|c|}{$60 \%$} \\
\hline & & $r$ & \multicolumn{9}{|c|}{ - } \\
\hline & & a & \multicolumn{9}{|c|}{ - } \\
\hline & \multirow{3}{*}{ Feb } & c & \multicolumn{9}{|c|}{$60 \%$} \\
\hline & & $r$ & \multicolumn{9}{|c|}{-} \\
\hline & & a & \multicolumn{9}{|c|}{-} \\
\hline & \multirow{3}{*}{ Mar } & c & \multicolumn{9}{|c|}{$60 \%$} \\
\hline & & $r$ & \multicolumn{9}{|c|}{ - } \\
\hline & & $a$ & \multicolumn{9}{|c|}{ - } \\
\hline \multirow{6}{*}{ 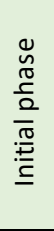 } & \multirow{3}{*}{ Apr } & c & \multicolumn{9}{|c|}{ - } \\
\hline & & $r$ & \multicolumn{9}{|c|}{$129 \%$} \\
\hline & & a & \multicolumn{9}{|c|}{$261 \%$} \\
\hline & \multirow{3}{*}{ May } & C & \multicolumn{9}{|c|}{ - } \\
\hline & & $r$ & \multicolumn{9}{|c|}{$129 \%$} \\
\hline & & a & & & & & $261 \%$ & & & & \\
\hline$\stackrel{100}{\subseteq}$ & & C & $60 \%$ & $104 \%$ & $149 \%$ & $193 \%$ & $237 \%$ & $282 \%$ & $326 \%$ & $371 \%$ & - \\
\hline 3 & Jun & $r$ & & & & & $129 \%$ & & & & \\
\hline ப் & & a & - & & & & & & & & \\
\hline & & c & & & & & - & & & & \\
\hline$\stackrel{\infty}{c}$ & Jul & $r$ & & & & & - & & & & \\
\hline Q & & $\mathrm{a}$ & & & & & $314 \%$ & & & & \\
\hline 르 & & c & & & & & - & & & & \\
\hline & Aug & $r$ & & & & & - & & & & \\
\hline & & a & $314 \%$ & $314 \%$ & $314 \%$ & $314 \%$ & $314 \%$ & $314 \%$ & $314 \%$ & $314 \%$ & $314 \%$ \\
\hline & & c & & & $60 \%$ & & & $149 \%$ & $237 \%$ & $326 \%$ & - \\
\hline & Sep & $r$ & & & & & - & & & & \\
\hline 荧 & & a & & & - & & . & & & & \\
\hline$\underset{0}{2}$ & & c & & & $60 \%$ & & & $149 \%$ & $237 \%$ & $326 \%$ & - \\
\hline$\Sigma$ & Oct & $r$ & & & & & - & & & & \\
\hline & & a & & & - & & & & & & \\
\hline & & c & & & & & $60 \%$ & & & & \\
\hline & Nov & $r$ & & & & & - & & & & \\
\hline$\stackrel{0}{\underline{4}}$ & & $\mathrm{a}$ & & & & & - & & & & \\
\hline$\frac{d}{\pi}$ & & C & & & & & $60 \%$ & & & & \\
\hline & Dec & $r$ & & & & & - & & & & \\
\hline & & a & & & & & - & & & & \\
\hline
\end{tabular}

Figure S.2. Po Plain case: relative damage (Eq. 2 in the paper) to maize crops (in case of conventional tillage) for different combinations of times of flood occurrence (i.e. month), flood intensities (i.e. water depth and flood duration) and damage alleviation strategies ("c"=continuation; "r"=reseeding; "a"=abandoning). Results for the " $\mathrm{r}$ " option are obtained by assuming a null yield penalty for late (re-)planting. 


\section{Wheat crops}

Table S.2. Average yield and price for wheat in the Province of Lodi over the last five years

\begin{tabular}{|c|c|}
\hline Yield $[\mathbf{q} / \mathbf{h a}]$ & 75 \\
\hline Price $[\boldsymbol{\epsilon} / \mathbf{q}]$ & 23 \\
\hline
\end{tabular}

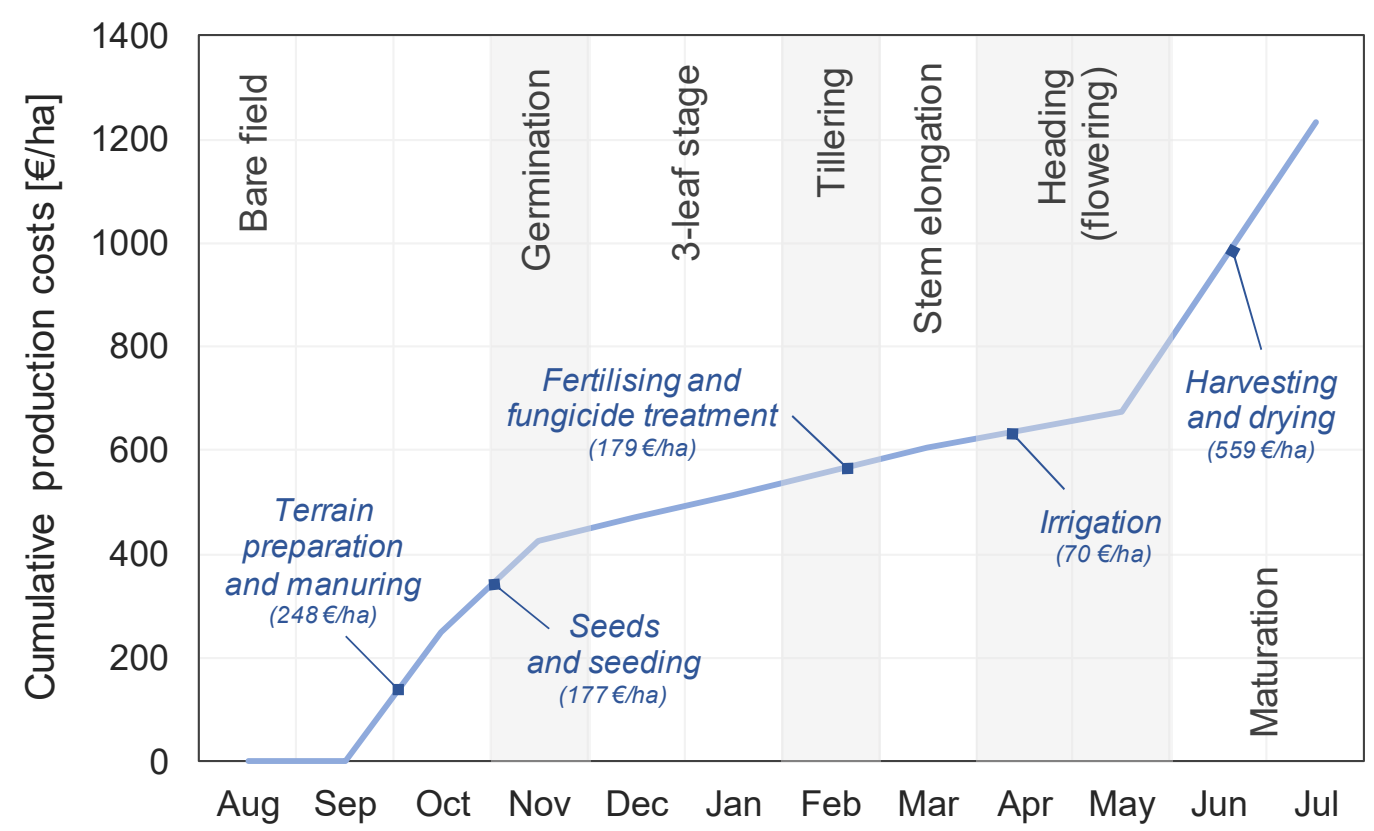

Figure S.3. Distribution of production costs over the year in the Scenario 0 for wheat crops, in case of minimum tillage

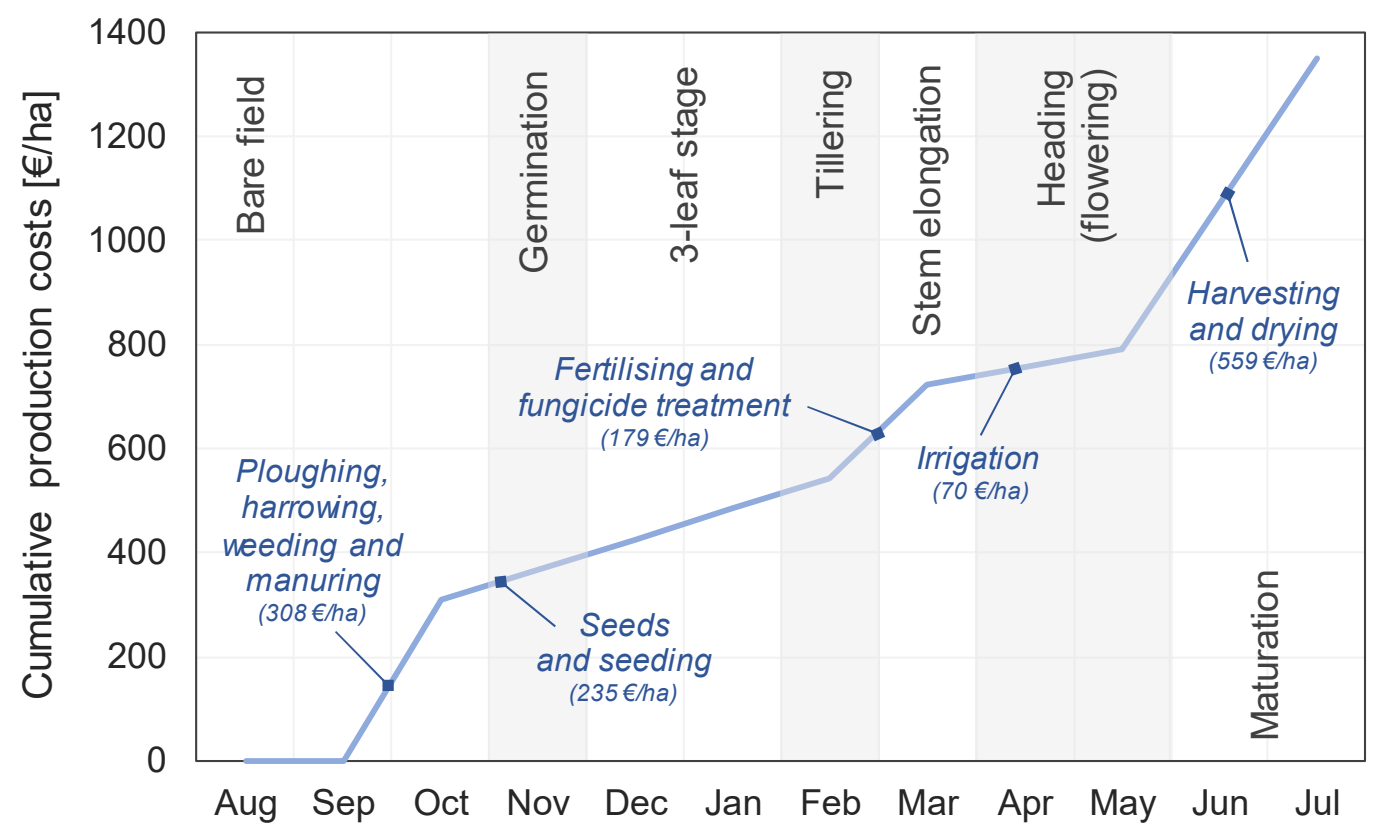

Figure S.4. Distribution of production costs over the year in the Scenario 0 for wheat crops, in case of conventional tillage 


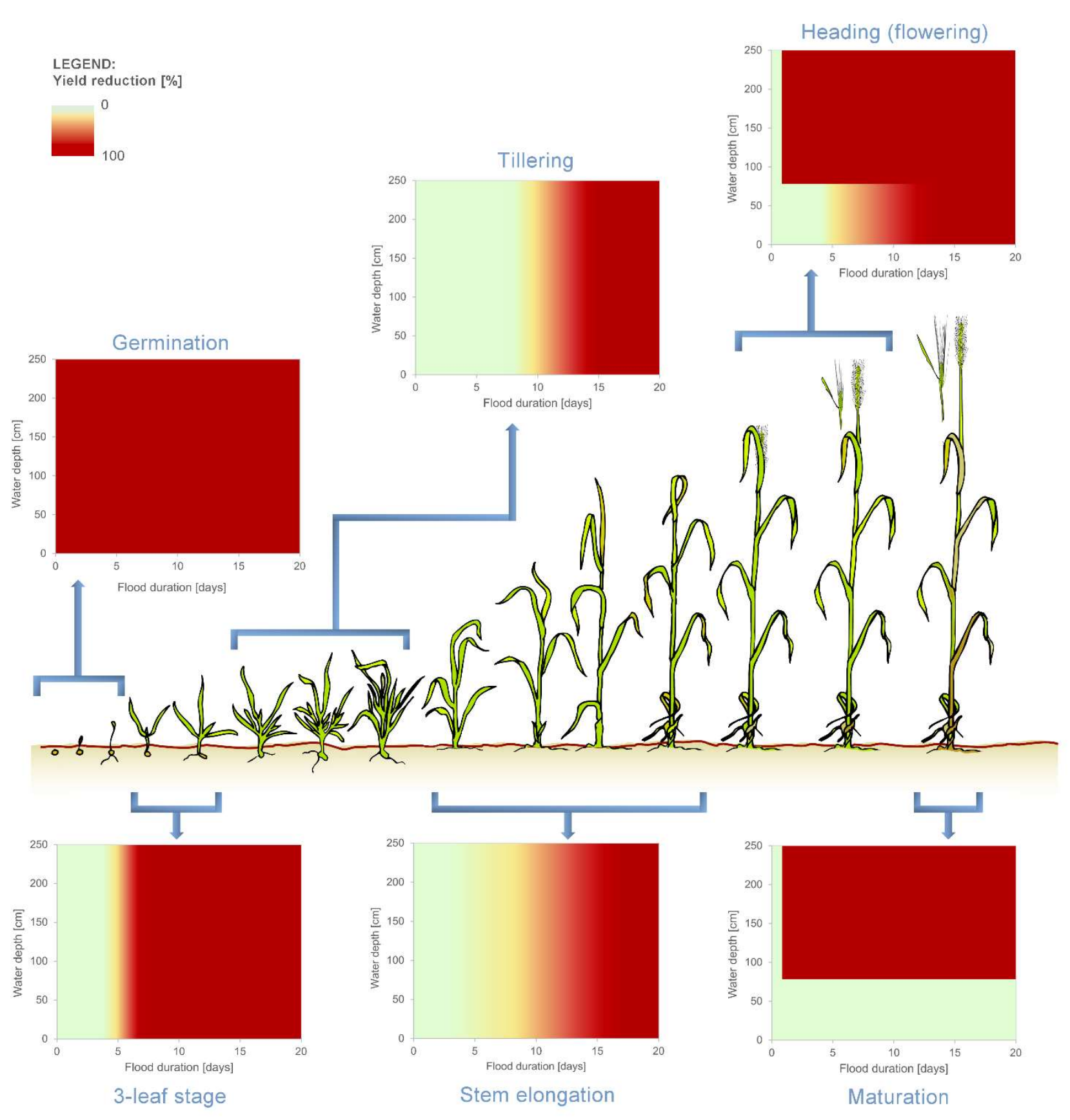

Figure S.5. Physical damage to wheat and barley as a function of vegetative stage, flood depth and duration (adapted from Agenais et al., 2013) 
Table S.3. Yield reduction and change in production costs on the basis of damage alleviation strategies for wheat crops, in the case of minimum tillage

\begin{tabular}{|c|c|c|c|c|c|c|c|}
\hline $\begin{array}{l}\text { Time of the } \\
\text { flood }\end{array}$ & $\begin{array}{l}\text { Vegetative } \\
\text { stage }\end{array}$ & $\begin{array}{c}\text { Alleviation } \\
\text { strategy }\end{array}$ & $\begin{array}{c}\text { Yield } \\
\text { reduction } \\
{[\%]}\end{array}$ & Additional costs & $€ / \mathbf{h a}$ & Avoided costs & €/ha \\
\hline $\begin{array}{l}\text { August - } \\
\text { October }\end{array}$ & Bare field & Continuation & 0 & $\begin{array}{l}\text { Soil restoring (sediment } \\
\text { removal and terrain levelling) }\end{array}$ & 500 & & \\
\hline \multirow{5}{*}{ November } & \multirow{5}{*}{ Germination } & \multirow{3}{*}{ Abandoning } & \multirow{3}{*}{100} & \multirow{3}{*}{$\begin{array}{l}\text { Soil restoring (sediment } \\
\text { removal and terrain levelling) }\end{array}$} & \multirow{3}{*}{500} & $\begin{array}{l}\text { Fertilising and fungicide } \\
\text { treatment }\end{array}$ & 179 \\
\hline & & & & & & Irrigation & 70 \\
\hline & & & & & & Harvesting and drying & 559 \\
\hline & & \multirow[t]{2}{*}{ Reseeding } & \multirow[t]{2}{*}{0} & $\begin{array}{l}\text { Soil restoring (sediment } \\
\text { removal and terrain levelling) }\end{array}$ & 500 & & \\
\hline & & & & Seeds and reseeding & 177 & & \\
\hline \multirow{6}{*}{$\begin{array}{l}\text { December - } \\
\text { January }\end{array}$} & \multirow{6}{*}{ 3-leaf stage } & Continuation & see Fig. A.5 & $\begin{array}{l}\text { Soil restoring (sediment } \\
\text { removal and terrain levelling) }\end{array}$ & 500 & & \\
\hline & & \multirow{3}{*}{ Abandoning } & \multirow{3}{*}{100} & \multirow{3}{*}{$\begin{array}{l}\text { Soil restoring (sediment } \\
\text { removal and terrain levelling) }\end{array}$} & \multirow{3}{*}{500} & $\begin{array}{l}\text { Fertilising and fungicide } \\
\text { treatment }\end{array}$ & 179 \\
\hline & & & & & & Irrigation & 70 \\
\hline & & & & & & Harvesting and drying & 559 \\
\hline & & \multirow[t]{2}{*}{ Reseeding } & \multirow[t]{2}{*}{0} & $\begin{array}{l}\text { Soil restoring (sediment } \\
\text { removal and terrain levelling) }\end{array}$ & 500 & & \\
\hline & & & & Seeds and reseeding & 177 & & \\
\hline \multirow{6}{*}{ February } & \multirow{6}{*}{ Tillering } & Continuation & see Fig. A.5 & $\begin{array}{l}\text { Soil restoring (sediment } \\
\text { removal and terrain levelling) }\end{array}$ & 500 & & \\
\hline & & \multirow{3}{*}{ Abandoning } & \multirow{3}{*}{100} & \multirow{3}{*}{$\begin{array}{l}\text { Soil restoring (sediment } \\
\text { removal and terrain levelling) }\end{array}$} & \multirow{3}{*}{500} & $\begin{array}{l}\text { Fertilising and fungicide } \\
\text { treatment }\end{array}$ & 179 \\
\hline & & & & & & Irrigation & 70 \\
\hline & & & & & & Harvesting and drying & 559 \\
\hline & & \multirow[t]{2}{*}{ Reseeding } & \multirow[t]{2}{*}{0} & $\begin{array}{l}\text { Soil restoring (sediment } \\
\text { removal and terrain levelling) }\end{array}$ & 500 & & \\
\hline & & & & Seeds and reseeding & 177 & & \\
\hline \multirow{3}{*}{ March } & \multirow{3}{*}{$\begin{array}{c}\text { Stem } \\
\text { elongation }\end{array}$} & Continuation & see Fig. A.5 & $\begin{array}{l}\text { Soil restoring (sediment } \\
\text { removal and terrain levelling) }\end{array}$ & 500 & & \\
\hline & & \multirow{2}{*}{ Abandoning } & \multirow{2}{*}{100} & \multirow{2}{*}{$\begin{array}{l}\text { Soil restoring (sediment } \\
\text { removal and terrain levelling) }\end{array}$} & \multirow{2}{*}{500} & Irrigation & 70 \\
\hline & & & & & & Harvesting and drying & 559 \\
\hline \multirow{2}{*}{ April - May } & \multirow{2}{*}{$\begin{array}{l}\text { Heading } \\
\text { (flowering) }\end{array}$} & Continuation & see Fig. A.5 & $\begin{array}{l}\text { Soil restoring (sediment } \\
\text { removal and terrain levelling) }\end{array}$ & 500 & & \\
\hline & & Abandoning & 100 & $\begin{array}{l}\text { Soil restoring (sediment } \\
\text { removal and terrain levelling) }\end{array}$ & 500 & Harvesting and drying & 559 \\
\hline June - Julv & Maturation & Continuation & see Fig. A.5 & $\begin{array}{l}\text { Soil restoring (sediment } \\
\text { removal and terrain levelling) }\end{array}$ & 500 & & \\
\hline June - July & phase & Abandoning & 100 & $\begin{array}{l}\text { Soil restoring (sediment } \\
\text { removal and terrain levelling) }\end{array}$ & 500 & Harvesting and drying & 559 \\
\hline
\end{tabular}


Table S.4. Yield reduction and change in production costs on the basis of damage alleviation strategies for wheat crops, in the case of conventional tillage

\begin{tabular}{|c|c|c|c|c|c|c|c|}
\hline $\begin{array}{l}\text { Time of the } \\
\text { flood }\end{array}$ & $\begin{array}{l}\text { Vegetative } \\
\text { stage }\end{array}$ & $\begin{array}{l}\text { Alleviation } \\
\text { strategy }\end{array}$ & $\begin{array}{c}\text { Yield } \\
\text { reduction } \\
{[\%]} \\
\end{array}$ & Additional costs & $€ / \mathbf{h a}$ & Avoided costs & $€ /$ ha \\
\hline $\begin{array}{l}\text { August - } \\
\text { October }\end{array}$ & Bare field & Continuation & 0 & $\begin{array}{l}\text { Soil restoring (sediment } \\
\text { removal and terrain levelling) }\end{array}$ & 500 & & \\
\hline \multirow{5}{*}{ November } & \multirow{5}{*}{ Germination } & \multirow{3}{*}{ Abandoning } & \multirow{3}{*}{100} & \multirow{3}{*}{$\begin{array}{l}\text { Soil restoring (sediment } \\
\text { removal and terrain levelling) }\end{array}$} & \multirow{3}{*}{500} & $\begin{array}{l}\text { Fertilising and fungicide } \\
\text { treatment }\end{array}$ & 179 \\
\hline & & & & & & Irrigation & 70 \\
\hline & & & & & & Harvesting and drying & 559 \\
\hline & & \multirow[t]{2}{*}{ Reseeding } & \multirow[t]{2}{*}{0} & $\begin{array}{l}\text { Soil restoring (sediment } \\
\text { removal and terrain levelling) }\end{array}$ & 500 & & \\
\hline & & & & Seeds and reseeding & 235 & & \\
\hline \multirow{6}{*}{$\begin{array}{l}\text { December - } \\
\text { January }\end{array}$} & \multirow{6}{*}{ 3-leaf stage } & Continuation & see Fig. A.5 & $\begin{array}{l}\text { Soil restoring (sediment } \\
\text { removal and terrain levelling) }\end{array}$ & 500 & & \\
\hline & & \multirow{3}{*}{ Abandoning } & \multirow{3}{*}{100} & \multirow{3}{*}{$\begin{array}{l}\text { Soil restoring (sediment } \\
\text { removal and terrain levelling) }\end{array}$} & \multirow{3}{*}{500} & $\begin{array}{l}\text { Fertilising and fungicide } \\
\text { treatment }\end{array}$ & 179 \\
\hline & & & & & & Irrigation & 70 \\
\hline & & & & & & Harvesting and drying & 559 \\
\hline & & \multirow[t]{2}{*}{ Reseeding } & \multirow[t]{2}{*}{0} & $\begin{array}{l}\text { Soil restoring (sediment } \\
\text { removal and terrain levelling) }\end{array}$ & 500 & & \\
\hline & & & & Seeds and reseeding & 235 & & \\
\hline \multirow{6}{*}{ February } & \multirow{6}{*}{ Tillering } & Continuation & see Fig. A.5 & $\begin{array}{l}\text { Soil restoring (sediment } \\
\text { removal and terrain levelling) }\end{array}$ & 500 & & \\
\hline & & \multirow{3}{*}{ Abandoning } & \multirow{3}{*}{100} & \multirow{3}{*}{$\begin{array}{l}\text { Soil restoring (sediment } \\
\text { removal and terrain levelling) }\end{array}$} & \multirow{3}{*}{500} & $\begin{array}{l}\text { Fertilising and fungicide } \\
\text { treatment }\end{array}$ & 179 \\
\hline & & & & & & Irrigation & 70 \\
\hline & & & & & & Harvesting and drying & 559 \\
\hline & & \multirow[t]{2}{*}{ Reseeding } & \multirow[t]{2}{*}{0} & $\begin{array}{l}\text { Soil restoring (sediment } \\
\text { removal and terrain levelling) }\end{array}$ & 500 & & \\
\hline & & & & Seeds and reseeding & 235 & & \\
\hline \multirow{3}{*}{ March } & \multirow{3}{*}{$\begin{array}{l}\text { Stem } \\
\text { elongation }\end{array}$} & Continuation & see Fig. A.5 & $\begin{array}{l}\text { Soil restoring (sediment } \\
\text { removal and terrain levelling) }\end{array}$ & 500 & & \\
\hline & & \multirow{2}{*}{ Abandoning } & \multirow{2}{*}{100} & \multirow{2}{*}{$\begin{array}{l}\text { Soil restoring (sediment } \\
\text { removal and terrain levelling) }\end{array}$} & \multirow{2}{*}{500} & Irrigation & 70 \\
\hline & & & & & & Harvesting and drying & 559 \\
\hline \multirow{2}{*}{ April - May } & \multirow{2}{*}{$\begin{array}{l}\text { Heading } \\
\text { (flowering) }\end{array}$} & Continuation & see Fig. A.5 & $\begin{array}{l}\text { Soil restoring (sediment } \\
\text { removal and terrain levelling) }\end{array}$ & 500 & & \\
\hline & & Abandoning & 100 & $\begin{array}{l}\text { Soil restoring (sediment } \\
\text { removal and terrain levelling) }\end{array}$ & 500 & Harvesting and drying & 559 \\
\hline June - Julv & Maturation & Continuation & see Fig. A.5 & $\begin{array}{l}\text { Soil restoring (sediment } \\
\text { removal and terrain levelling) }\end{array}$ & 500 & & \\
\hline June - July & phase & Abandoning & 100 & $\begin{array}{l}\text { Soil restoring (sediment } \\
\text { removal and terrain levelling) }\end{array}$ & 500 & Harvesting and drying & 559 \\
\hline
\end{tabular}



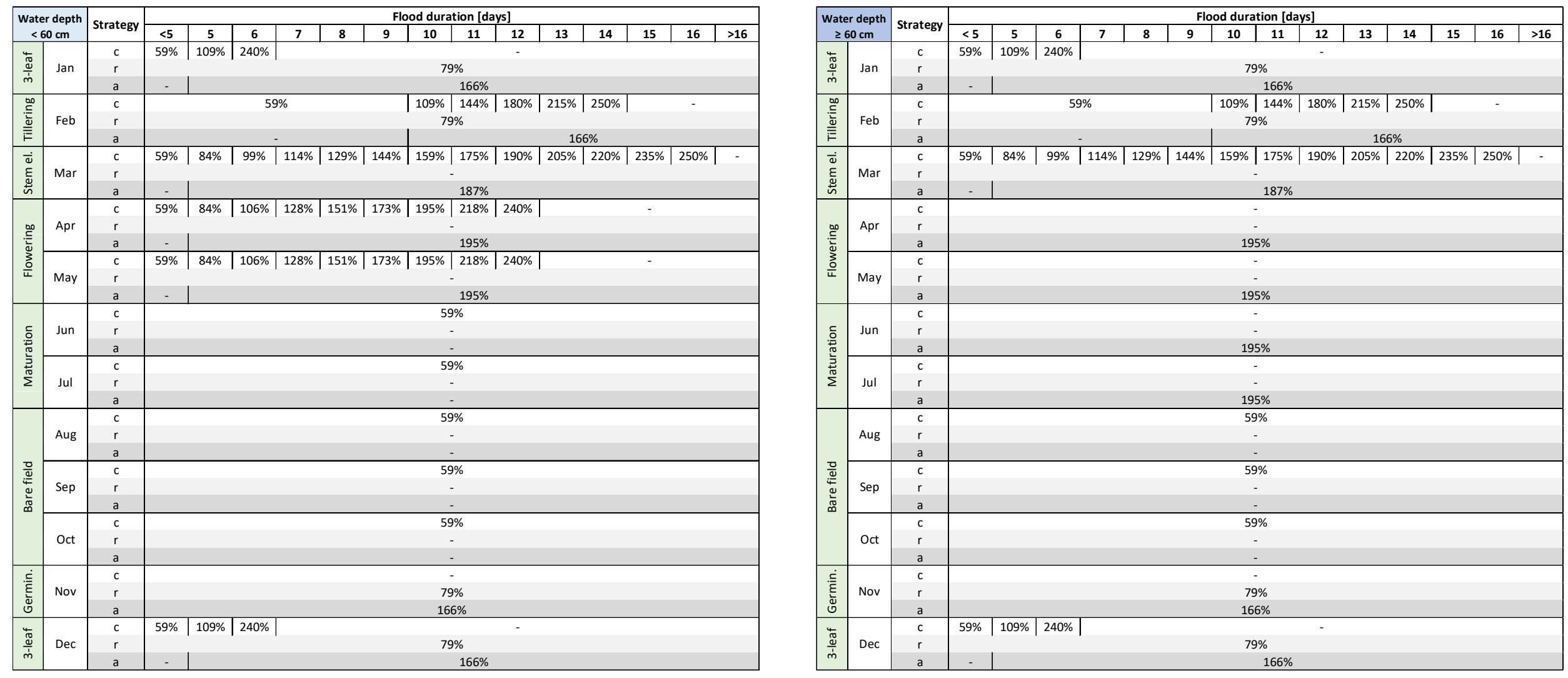

Figure S.6. Po Plain case: relative damage (Eq. 2 in the paper) to wheat crops (in case of minimum tillage) for different combinations of times of flood occurrence (i.e. month), flood intensities (i.e. water depth and flood duration) and damage alleviation strategies ("c"=continuation; "r"=reseeding; "a"=abandoning). Results for the " $r$ " option are obtained by assuming a null yield penalty for late (re-)planting. 

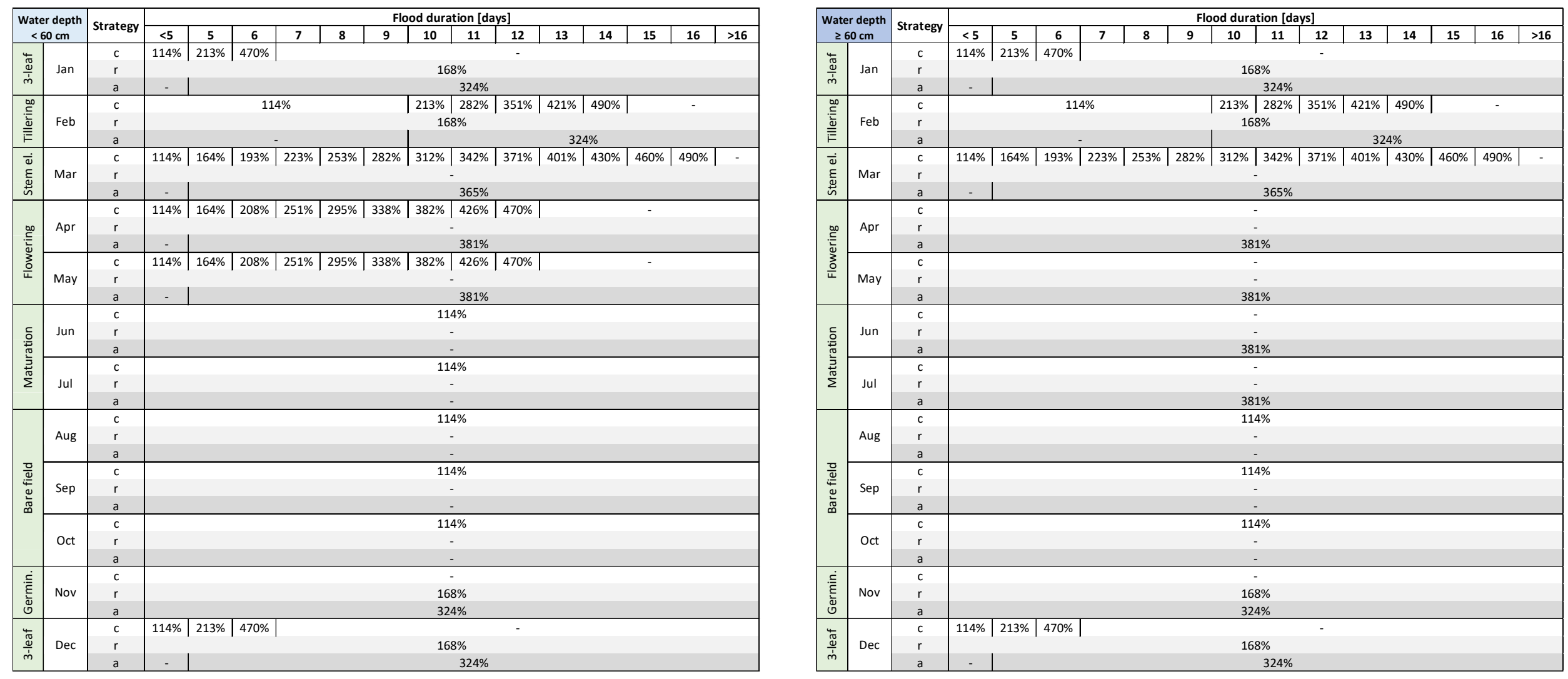

Figure S.7. Po Plain case: relative damage (Eq. 2 in the paper) to wheat crops (in case of conventional tillage) for different combinations of times of flood occurrence (i.e. month), flood intensities (i.e. water depth and flood duration) and damage alleviation strategies ("c"=continuation; "r"=reseeding; "a"=abandoning). Results for the " $r$ " option are obtained by assuming a null yield penalty for late (re-)planting. 


\section{Barley crops}

Table S.5. Average yield and price for barley in the Province of Lodi over the last five years

\begin{tabular}{|c|c|}
\hline Yield $[\mathbf{q} / \mathbf{h a}]$ & 70 \\
\hline Price $[\boldsymbol{\epsilon} / \mathbf{q}]$ & 19 \\
\hline
\end{tabular}

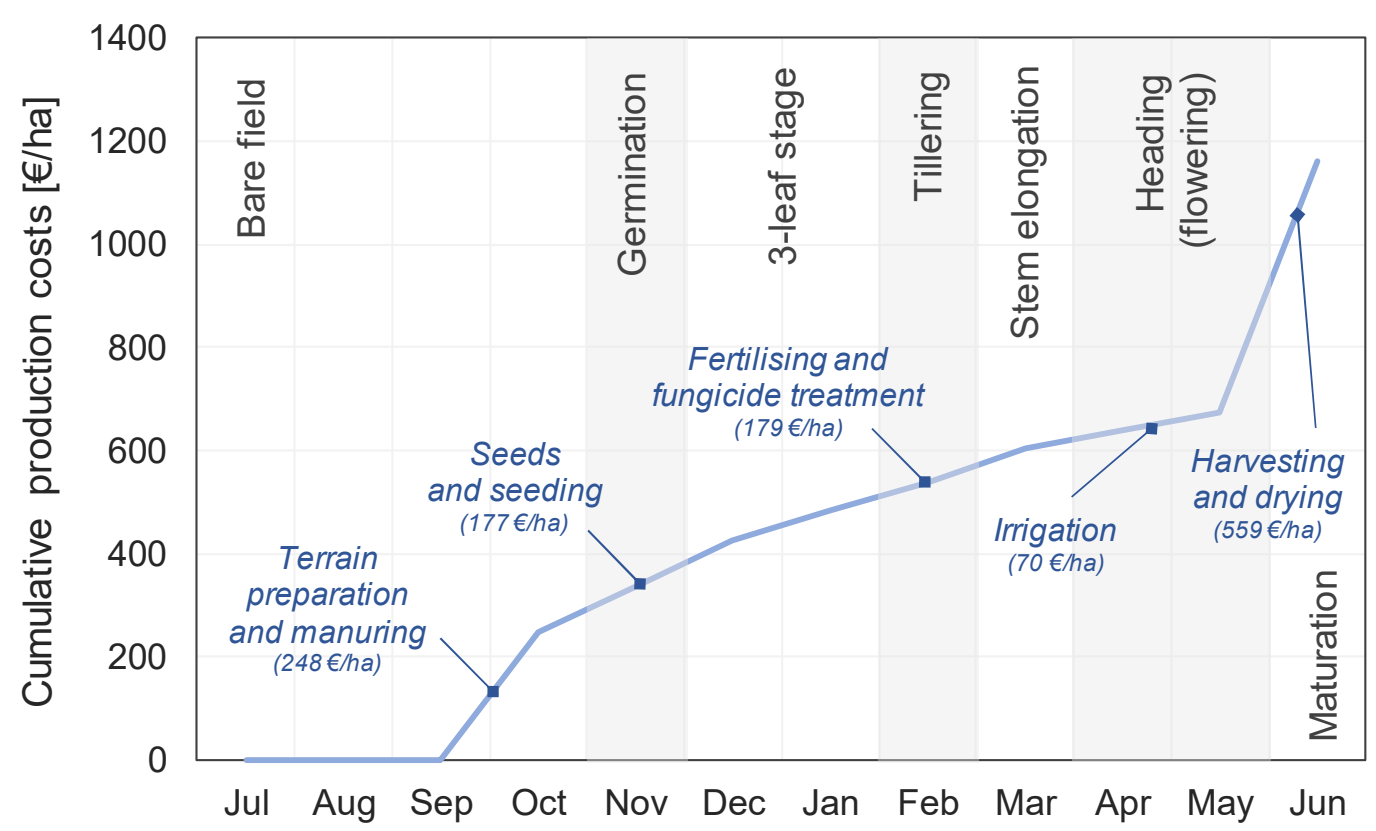

Figure S.8. Distribution of production costs over the year in the Scenario 0 for barley crops, in case of minimum tillage

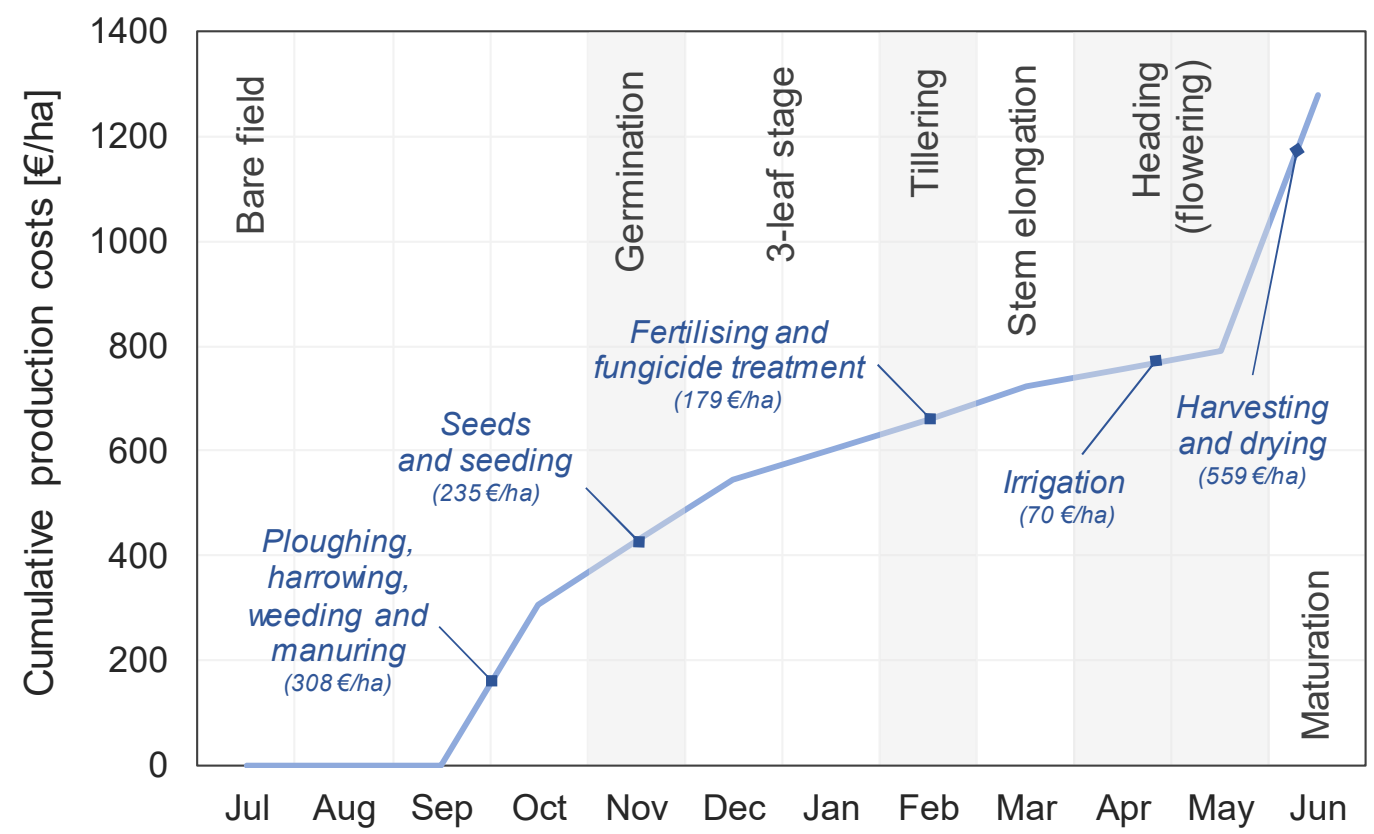

Figure S.9. Distribution of production costs over the year in the Scenario 0 for barley crops, in case of conventional tillage 
Table S.6. Yield reduction and change in production costs on the basis of damage alleviation strategies for barley crops, in the case of minimum tillage

\begin{tabular}{|c|c|c|c|c|c|c|c|}
\hline $\begin{array}{l}\text { Time of the } \\
\text { flood }\end{array}$ & $\begin{array}{l}\text { Vegetative } \\
\text { stage }\end{array}$ & $\begin{array}{l}\text { Alleviation } \\
\text { strategy }\end{array}$ & $\begin{array}{c}\text { Yield } \\
\text { reduction } \\
{[\%]}\end{array}$ & Additional costs & $€ / \mathbf{h a}$ & Avoided costs & $€ / \mathbf{h a}$ \\
\hline $\begin{array}{l}\text { July - } \\
\text { October }\end{array}$ & Bare field & Continuation & 0 & $\begin{array}{l}\text { Soil restoring (sediment } \\
\text { removal and terrain levelling) }\end{array}$ & 500 & & \\
\hline \multirow{5}{*}{ November } & \multirow{5}{*}{ Germination } & \multirow{3}{*}{ Abandoning } & \multirow{3}{*}{100} & \multirow{3}{*}{$\begin{array}{l}\text { Soil restoring (sediment } \\
\text { removal and terrain levelling) }\end{array}$} & \multirow{3}{*}{500} & $\begin{array}{l}\text { Fertilising and fungicide } \\
\text { treatment }\end{array}$ & 179 \\
\hline & & & & & & Irrigation & 70 \\
\hline & & & & & & Harvesting and drying & 559 \\
\hline & & Reseeding & 0 & $\begin{array}{l}\text { Soil restoring (sediment } \\
\text { removal and terrain levelling) }\end{array}$ & 500 & & \\
\hline & & & & Seeds and reseeding & 177 & & \\
\hline \multirow{6}{*}{$\begin{array}{l}\text { December - } \\
\text { January }\end{array}$} & \multirow{6}{*}{ 3-leaf stage } & Continuation & see Fig. A.5 & $\begin{array}{l}\text { Soil restoring (sediment } \\
\text { removal and terrain levelling) }\end{array}$ & 500 & & \\
\hline & & \multirow{3}{*}{ Abandoning } & \multirow{3}{*}{100} & \multirow{3}{*}{$\begin{array}{l}\text { Soil restoring (sediment } \\
\text { removal and terrain levelling) }\end{array}$} & \multirow{3}{*}{500} & $\begin{array}{l}\text { Fertilising and fungicide } \\
\text { treatment }\end{array}$ & 179 \\
\hline & & & & & & Irrigation & 70 \\
\hline & & & & & & Harvesting and drying & 559 \\
\hline & & \multirow[t]{2}{*}{ Reseeding } & \multirow[t]{2}{*}{0} & $\begin{array}{l}\text { Soil restoring (sediment } \\
\text { removal and terrain levelling) }\end{array}$ & 500 & & \\
\hline & & & & Seeds and reseeding & 177 & & \\
\hline \multirow{6}{*}{ February } & \multirow{6}{*}{ Tillering } & Continuation & see Fig. A.5 & $\begin{array}{l}\text { Soil restoring (sediment } \\
\text { removal and terrain levelling) }\end{array}$ & 500 & & \\
\hline & & \multirow{3}{*}{ Abandoning } & \multirow{3}{*}{100} & \multirow{3}{*}{$\begin{array}{l}\text { Soil restoring (sediment } \\
\text { removal and terrain levelling) }\end{array}$} & \multirow{3}{*}{500} & $\begin{array}{l}\text { Fertilising and fungicide } \\
\text { treatment }\end{array}$ & 179 \\
\hline & & & & & & Irrigation & 70 \\
\hline & & & & & & Harvesting and drying & 559 \\
\hline & & \multirow[t]{2}{*}{ Reseeding } & \multirow[t]{2}{*}{0} & $\begin{array}{l}\text { Soil restoring (sediment } \\
\text { removal and terrain levelling) }\end{array}$ & 500 & & \\
\hline & & & & Seeds and reseeding & 177 & & \\
\hline \multirow{3}{*}{ March } & \multirow{3}{*}{$\begin{array}{l}\text { Stem } \\
\text { elongation }\end{array}$} & Continuation & see Fig. A.5 & $\begin{array}{l}\text { Soil restoring (sediment } \\
\text { removal and terrain levelling) }\end{array}$ & 500 & & \\
\hline & & \multirow{2}{*}{ Abandoning } & \multirow{2}{*}{100} & \multirow{2}{*}{$\begin{array}{l}\text { Soil restoring (sediment } \\
\text { removal and terrain levelling) }\end{array}$} & \multirow{2}{*}{500} & Irrigation & 70 \\
\hline & & & & & & Harvesting and drying & 559 \\
\hline \multirow{2}{*}{ April - May } & \multirow{2}{*}{$\begin{array}{l}\text { Heading } \\
\text { (flowering) }\end{array}$} & Continuation & see Fig. A.5 & $\begin{array}{l}\text { Soil restoring (sediment } \\
\text { removal and terrain levelling) }\end{array}$ & 500 & & \\
\hline & & Abandoning & 100 & $\begin{array}{l}\text { Soil restoring (sediment } \\
\text { removal and terrain levelling) }\end{array}$ & 500 & Harvesting and drying & 559 \\
\hline \multirow{2}{*}{ June } & \multirow{2}{*}{$\begin{array}{l}\text { Maturation } \\
\text { phase }\end{array}$} & Continuation & see Fig. A.5 & $\begin{array}{l}\text { Soil restoring (sediment } \\
\text { removal and terrain levelling) }\end{array}$ & 500 & & \\
\hline & & Abandoning & 100 & $\begin{array}{l}\text { Soil restoring (sediment } \\
\text { removal and terrain levelling) }\end{array}$ & 500 & Harvesting and drying & 559 \\
\hline
\end{tabular}


Table S.7. Yield reduction and change in production costs on the basis of damage alleviation strategies for barley crops, in the case of conventional tillage

\begin{tabular}{|c|c|c|c|c|c|c|c|}
\hline $\begin{array}{l}\text { Time of the } \\
\text { flood }\end{array}$ & $\begin{array}{l}\text { Vegetative } \\
\text { stage }\end{array}$ & $\begin{array}{l}\text { Alleviation } \\
\text { strategy }\end{array}$ & $\begin{array}{c}\text { Yield } \\
\text { reduction } \\
{[\%]}\end{array}$ & Additional costs & $€ / \mathbf{h a}$ & Avoided costs & $€ / \mathbf{h a}$ \\
\hline $\begin{array}{l}\text { July - } \\
\text { October }\end{array}$ & Bare field & Continuation & 0 & $\begin{array}{l}\text { Soil restoring (sediment } \\
\text { removal and terrain levelling) }\end{array}$ & 500 & & \\
\hline \multirow{5}{*}{ November } & \multirow{5}{*}{ Germination } & \multirow{3}{*}{ Abandoning } & \multirow{3}{*}{100} & \multirow{3}{*}{$\begin{array}{l}\text { Soil restoring (sediment } \\
\text { removal and terrain levelling) }\end{array}$} & \multirow{3}{*}{500} & $\begin{array}{l}\text { Fertilising and fungicide } \\
\text { treatment }\end{array}$ & 179 \\
\hline & & & & & & Irrigation & 70 \\
\hline & & & & & & Harvesting and drying & 559 \\
\hline & & Reseeding & 0 & $\begin{array}{l}\text { Soil restoring (sediment } \\
\text { removal and terrain levelling) }\end{array}$ & 500 & & \\
\hline & & & & Seeds and reseeding & 235 & & \\
\hline \multirow{6}{*}{$\begin{array}{l}\text { December - } \\
\text { January }\end{array}$} & \multirow{6}{*}{ 3-leaf stage } & Continuation & see Fig. A.5 & $\begin{array}{l}\text { Soil restoring (sediment } \\
\text { removal and terrain levelling) }\end{array}$ & 500 & & \\
\hline & & \multirow{3}{*}{ Abandoning } & \multirow{3}{*}{100} & \multirow{3}{*}{$\begin{array}{l}\text { Soil restoring (sediment } \\
\text { removal and terrain levelling) }\end{array}$} & \multirow{3}{*}{500} & $\begin{array}{l}\text { Fertilising and fungicide } \\
\text { treatment }\end{array}$ & 179 \\
\hline & & & & & & Irrigation & 70 \\
\hline & & & & & & Harvesting and drying & 559 \\
\hline & & \multirow[t]{2}{*}{ Reseeding } & \multirow[t]{2}{*}{0} & $\begin{array}{l}\text { Soil restoring (sediment } \\
\text { removal and terrain levelling) }\end{array}$ & 500 & & \\
\hline & & & & Seeds and reseeding & 235 & & \\
\hline \multirow{6}{*}{ February } & \multirow{6}{*}{ Tillering } & Continuation & see Fig. A.5 & $\begin{array}{l}\text { Soil restoring (sediment } \\
\text { removal and terrain levelling) }\end{array}$ & 500 & & \\
\hline & & \multirow{3}{*}{ Abandoning } & \multirow{3}{*}{100} & \multirow{3}{*}{$\begin{array}{l}\text { Soil restoring (sediment } \\
\text { removal and terrain levelling) }\end{array}$} & \multirow{3}{*}{500} & $\begin{array}{l}\text { Fertilising and fungicide } \\
\text { treatment }\end{array}$ & 179 \\
\hline & & & & & & Irrigation & 70 \\
\hline & & & & & & Harvesting and drying & 559 \\
\hline & & \multirow[t]{2}{*}{ Reseeding } & \multirow[t]{2}{*}{0} & $\begin{array}{l}\text { Soil restoring (sediment } \\
\text { removal and terrain levelling) }\end{array}$ & 500 & & \\
\hline & & & & Seeds and reseeding & 235 & & \\
\hline \multirow{3}{*}{ March } & \multirow{3}{*}{$\begin{array}{l}\text { Stem } \\
\text { elongation }\end{array}$} & Continuation & see Fig. A.5 & $\begin{array}{l}\text { Soil restoring (sediment } \\
\text { removal and terrain levelling) }\end{array}$ & 500 & & \\
\hline & & \multirow{2}{*}{ Abandoning } & \multirow{2}{*}{100} & \multirow{2}{*}{$\begin{array}{l}\text { Soil restoring (sediment } \\
\text { removal and terrain levelling) }\end{array}$} & \multirow{2}{*}{500} & Irrigation & 70 \\
\hline & & & & & & Harvesting and drying & 559 \\
\hline \multirow{2}{*}{ April - May } & \multirow{2}{*}{$\begin{array}{l}\text { Heading } \\
\text { (flowering) }\end{array}$} & Continuation & see Fig. A.5 & $\begin{array}{l}\text { Soil restoring (sediment } \\
\text { removal and terrain levelling) }\end{array}$ & 500 & & \\
\hline & & Abandoning & 100 & $\begin{array}{l}\text { Soil restoring (sediment } \\
\text { removal and terrain levelling) }\end{array}$ & 500 & Harvesting and drying & 559 \\
\hline \multirow{2}{*}{ June } & \multirow{2}{*}{$\begin{array}{l}\text { Maturation } \\
\text { phase }\end{array}$} & Continuation & see Fig. A.5 & $\begin{array}{l}\text { Soil restoring (sediment } \\
\text { removal and terrain levelling) }\end{array}$ & 500 & & \\
\hline & & Abandoning & 100 & $\begin{array}{l}\text { Soil restoring (sediment } \\
\text { removal and terrain levelling) }\end{array}$ & 500 & Harvesting and drying & 559 \\
\hline
\end{tabular}




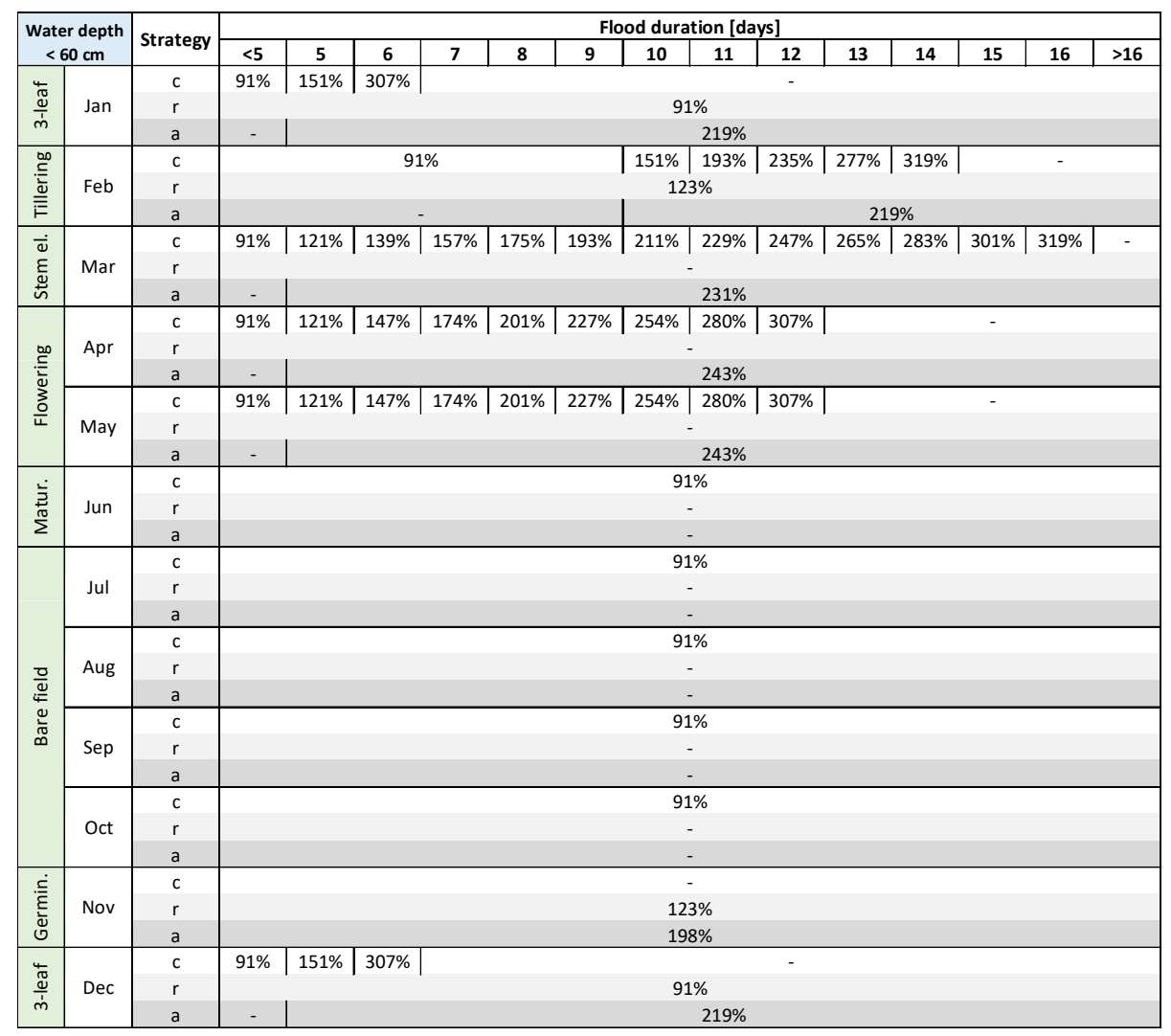

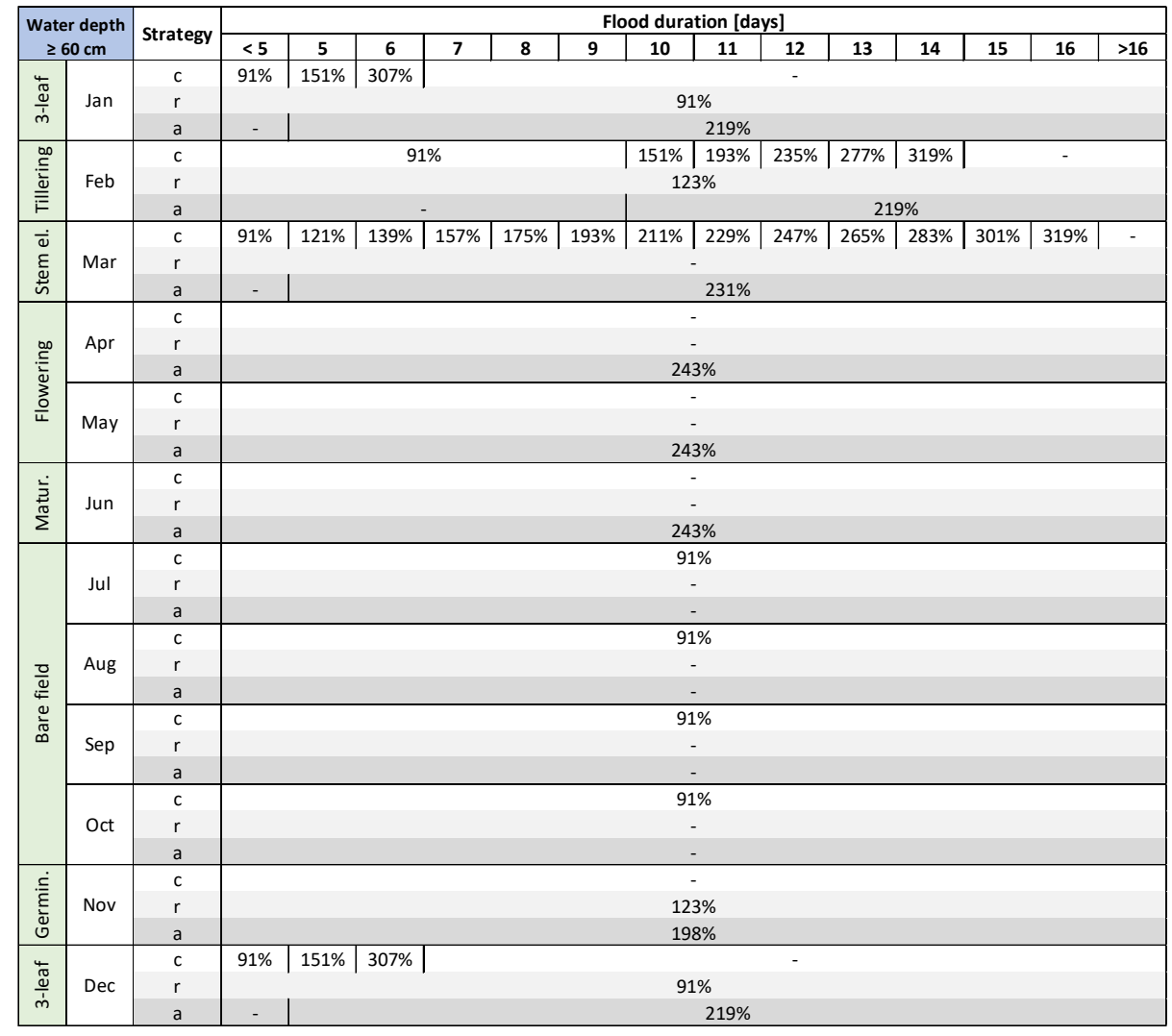

Figure S.10. Po Plain case: relative damage (Eq. 2 in the paper) to barley crops (in case of minimum tillage) for different combinations of times of flood occurrence (i.e. month), flood intensities (i.e. water depth and flood duration) and damage alleviation strategies ("c"=continuation; "r"=reseeding; "a"=abandoning). Results for the " $r$ " option are obtained by assuming a null yield penalty for late (re-)planting. 


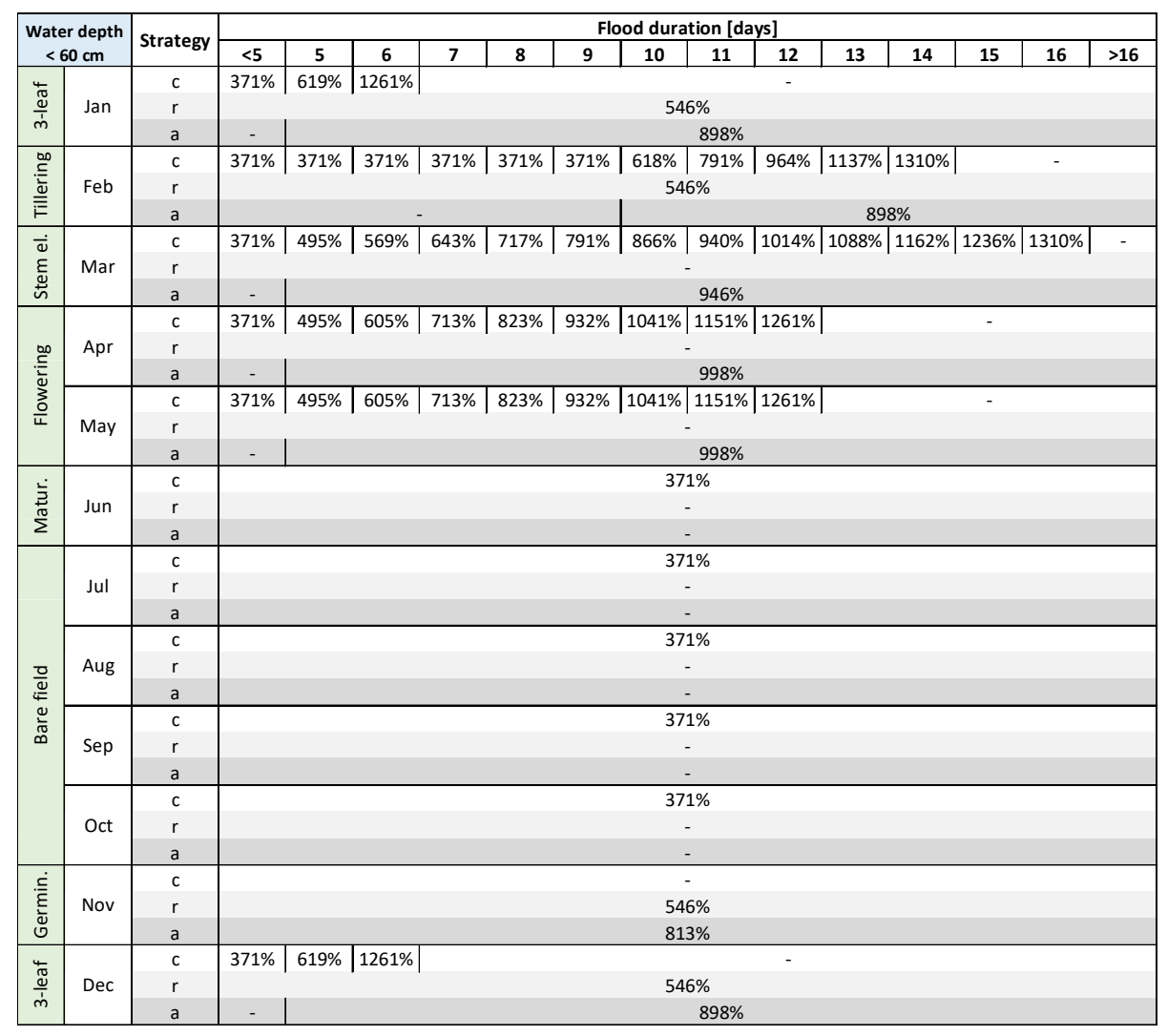

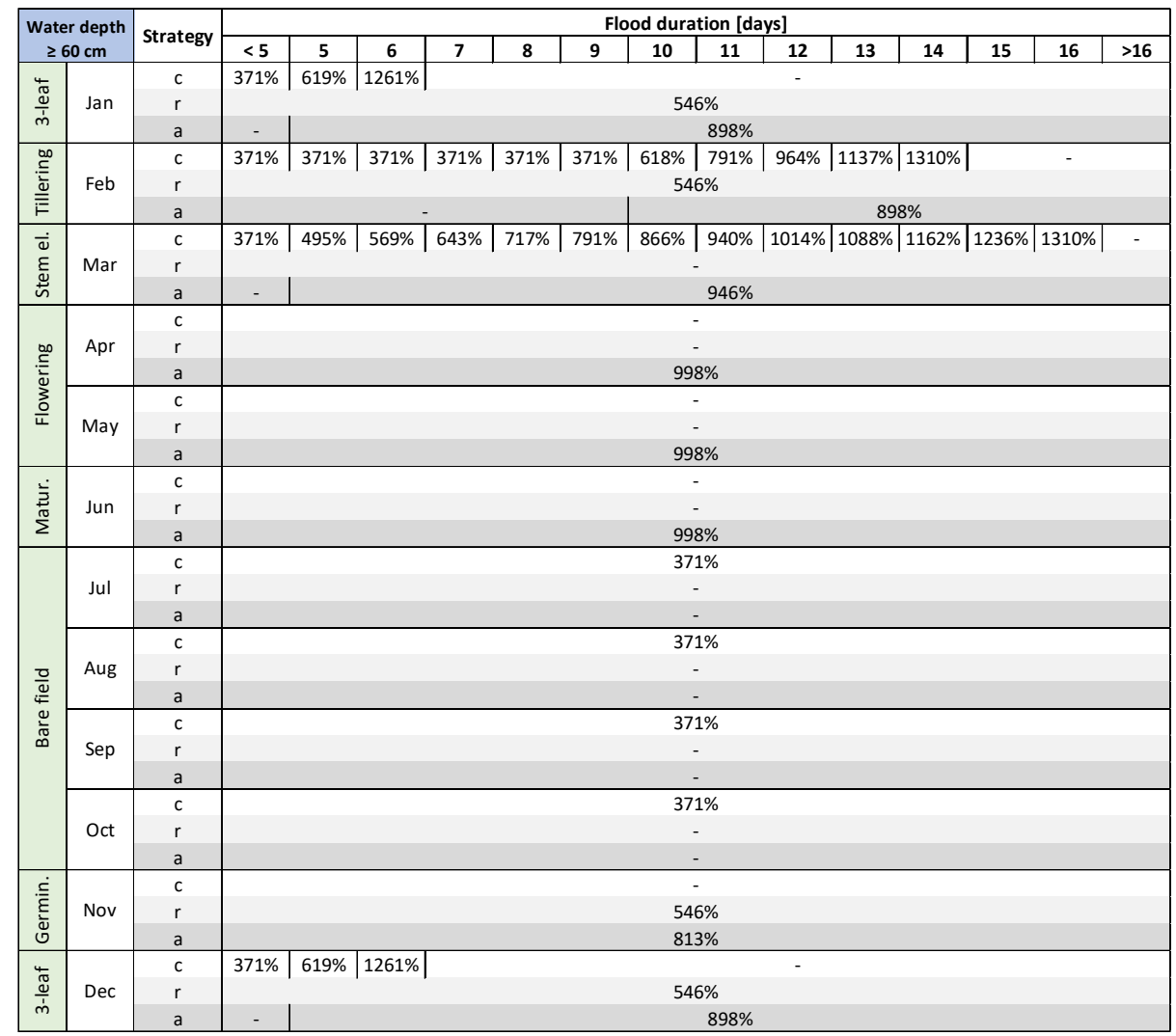

Figure S.11. Po Plain case: relative damage (Eq. 2 in the paper) to barley crops (in case of conventional tillage) for different combinations of times of occurrence of the flood (i.e. month), flood intensities (i.e. water depth and flood duration) and damage alleviation strategies ("c"=continuation; " $r$ "=reseeding; "a"=abandoning). Results for the "r" option are obtained by assuming a null yield penalty for late (re-)planting. 


\section{Grassland}

Table S.8. Average yield and price for grassland in the Province of Lodi over the last five years

\begin{tabular}{|c|c|c|c|c|c|}
\hline & Tot. & I mow. & II mow. & III mow. & IV mow. \\
\hline Yield [q/ha] & 120 & 60 & 20 & 20 & 20 \\
\hline Price [€/q] & \multicolumn{5}{|c|}{15.4} \\
\hline
\end{tabular}

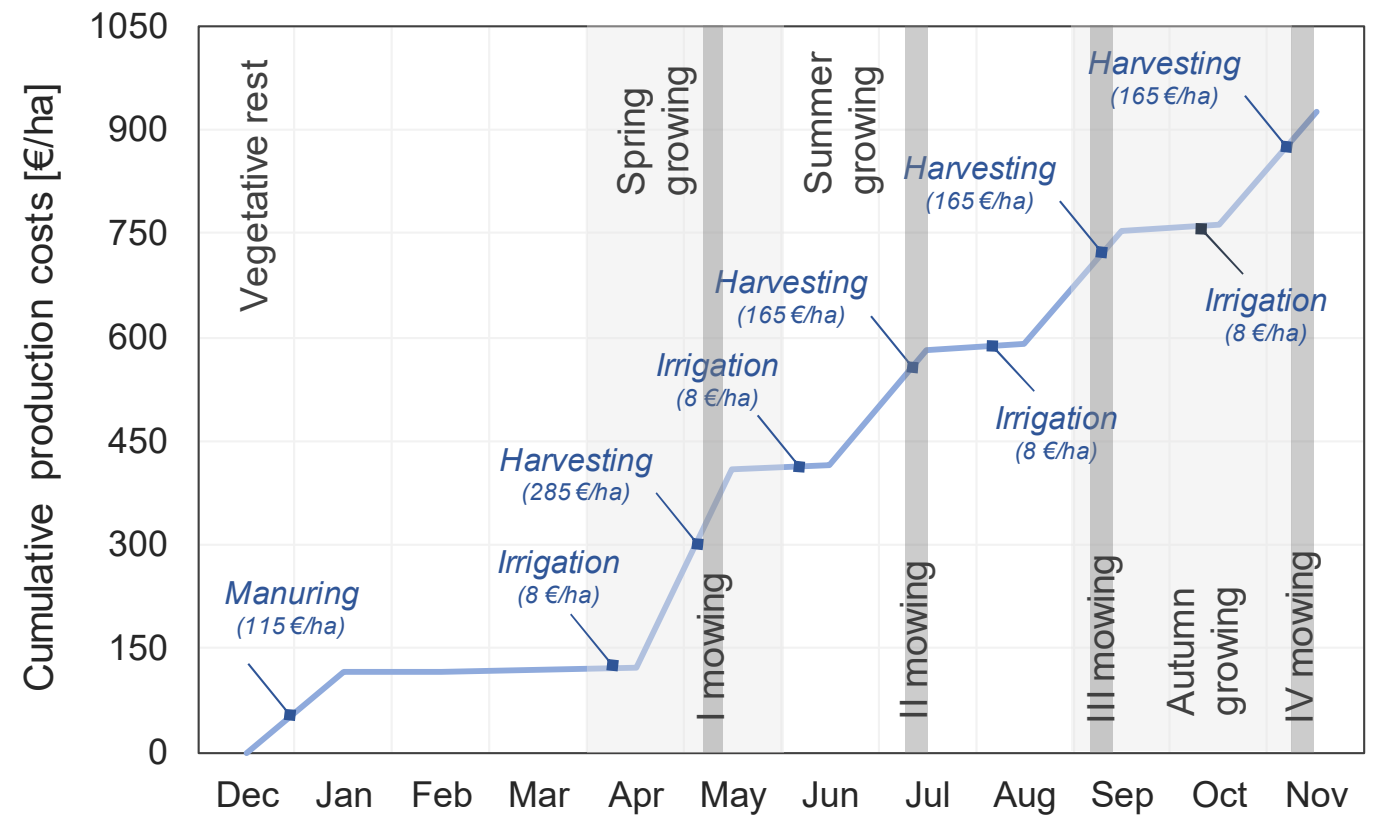

Figure S.12. Distribution of production costs over the year in the Scenario 0 for grassland

Assumptions for calculation of flood damage to grassland:

- Abandoning is not considered among the different alleviation strategies, as grassland is a perennial crop (i.e., if production is abandoned, the flood will impact on farmer's revenues also in the following years);

- The impact of the flood has influence only on the forthcoming harvest and not on successive ones;

- In case of flood, a reduction in the selling price is expected due to a lower quality harvest, as a function of flood duration and days remaining to the forthcoming harvest (see Figure A.14). 

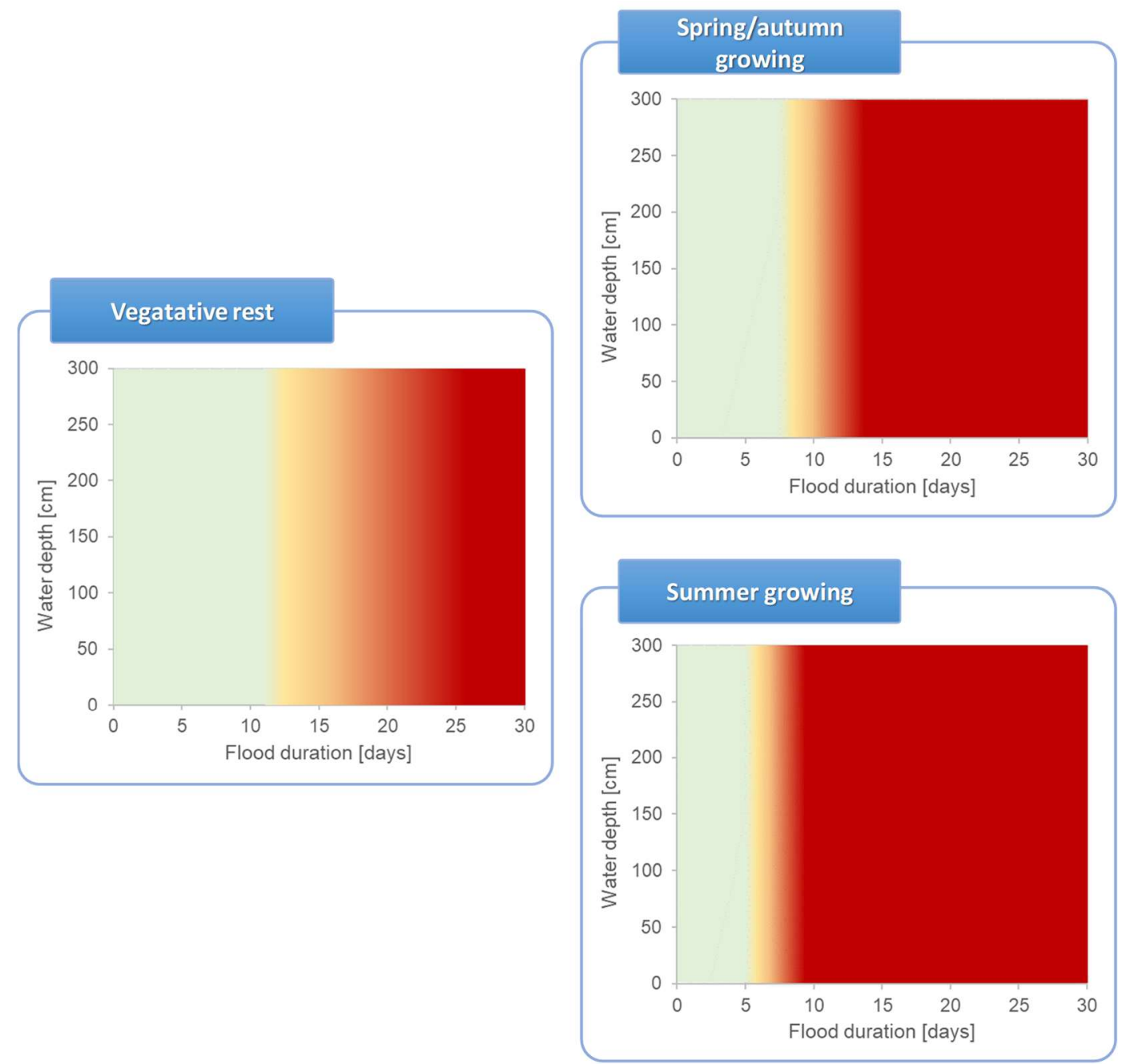

Figure S.13. Physical damage to grassland as a function of vegetative stage, flood depth and duration (adapted from Agenais et al., 2013)

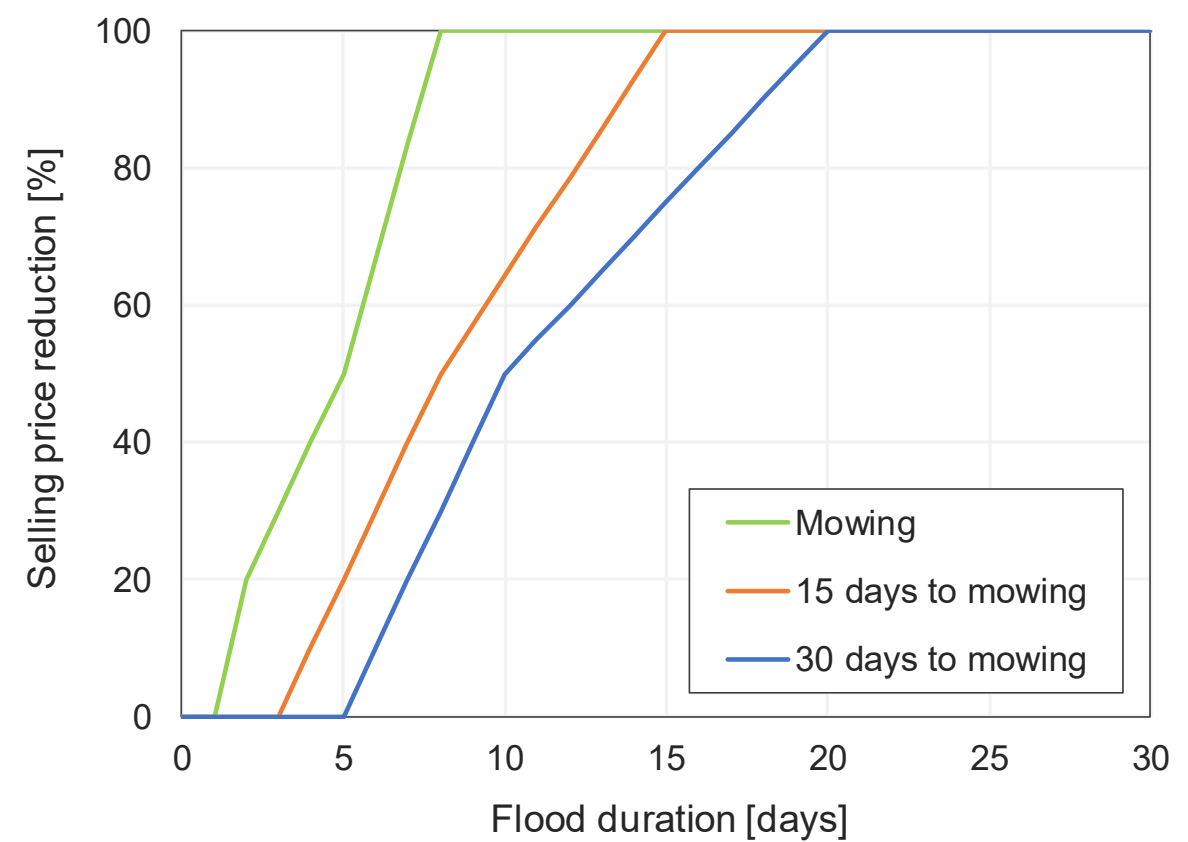

Figure S.14. Selling price reduction for different times of flood occurrence: mowing, 15 days to mowing and 30 days to mowing (adapted from Agenais et al., 2013) 
Table S.11. Yield reduction and change in production costs on the basis of damage alleviation strategies for grassland

\begin{tabular}{|c|c|c|c|c|c|c|c|}
\hline $\begin{array}{l}\text { Time of the } \\
\text { flood }\end{array}$ & $\begin{array}{l}\text { Vegetative } \\
\text { stage }\end{array}$ & $\begin{array}{l}\text { Alleviation } \\
\text { strategy }\end{array}$ & $\begin{array}{c}\text { Yield } \\
\text { reduction } \\
{[\%]}\end{array}$ & Additional costs & $€ /$ ha & Avoided costs & $€ /$ ha \\
\hline \multirow{3}{*}{$\begin{array}{l}\text { November - } \\
\text { March }\end{array}$} & \multirow{3}{*}{$\begin{array}{l}\text { Vegetative } \\
\text { rest }\end{array}$} & Continuation & $\begin{array}{l}\text { see Fig. } \\
\text { A.13 }\end{array}$ & $\begin{array}{l}\text { Soil restoring (sediment } \\
\text { removal and terrain levelling) }\end{array}$ & 500 & & \\
\hline & & \multirow{2}{*}{ Reseeding } & \multirow[t]{2}{*}{0} & $\begin{array}{l}\text { Soil restoring (sediment } \\
\text { removal and terrain levelling) }\end{array}$ & 500 & & \\
\hline & & & & Seeds and reseeding & 89 & & \\
\hline \multirow{3}{*}{ April - May } & \multirow{3}{*}{$\begin{array}{l}\text { Spring } \\
\text { growing }\end{array}$} & Continuation & $\begin{array}{l}\text { see Fig. } \\
\text { A. } 13\end{array}$ & & & & \\
\hline & & Reseeding & Only $1^{\text {st }}$ & $\begin{array}{l}\text { Soil restoring (sediment } \\
\text { removal and terrain levelling) }\end{array}$ & 500 & \multirow{2}{*}{$\begin{array}{l}\text { I mowing (if the flood } \\
\text { occurs before it) }\end{array}$} & \multirow[t]{2}{*}{285} \\
\hline & & & & Seeds and reseeding & 89 & & \\
\hline \multirow{4}{*}{ June - August } & \multirow{4}{*}{$\begin{array}{l}\text { Summer } \\
\text { growing }\end{array}$} & Continuation & $\begin{array}{l}\text { see Fig. } \\
\text { A. } 13\end{array}$ & & & & \\
\hline & & \multirow{3}{*}{ Reseeding } & \multirow{3}{*}{$\begin{array}{c}2^{\text {nd }} \text { or } 3^{\text {rd }} \\
\text { harvest lost }\end{array}$} & \multirow{2}{*}{$\begin{array}{l}\text { Soil restoring (sediment } \\
\text { removal and terrain levelling) }\end{array}$} & \multirow[t]{2}{*}{500} & $\begin{array}{l}\text { II mowing (if the flood } \\
\text { occurs before it) }\end{array}$ & 165 \\
\hline & & & & & & \multirow{2}{*}{$\begin{array}{l}\text { or III mowing (if the flood } \\
\text { occurs before it) }\end{array}$} & \multirow{2}{*}{165} \\
\hline & & & & Seeds and reseeding & 89 & & \\
\hline \multirow{3}{*}{$\begin{array}{l}\text { September - } \\
\text { October }\end{array}$} & \multirow{3}{*}{$\begin{array}{l}\text { Autumn } \\
\text { growing }\end{array}$} & Continuation & $\begin{array}{l}\text { see Fig. } \\
\text { A. } 13\end{array}$ & & & & \\
\hline & & Reseeding & Only $4^{\text {th }}$ & $\begin{array}{l}\text { Soil restoring (sediment } \\
\text { removal and terrain levelling) }\end{array}$ & 500 & \multirow{2}{*}{$\begin{array}{l}\text { IV mowing (if the flood } \\
\text { occurs before it) }\end{array}$} & \multirow[t]{2}{*}{165} \\
\hline & & & harvest lost & Seeds and reseeding & 89 & & \\
\hline
\end{tabular}

\begin{tabular}{|c|c|c|c|c|c|c|c|c|c|c|c|c|c|}
\hline & & \multirow{2}{*}{ Strategy } & \multicolumn{11}{|c|}{ Flood duration [days] } \\
\hline & & & $<5$ & 7 & 9 & 11 & 13 & 15 & 17 & 19 & 21 & 23 & $>25$ \\
\hline \multirow{6}{*}{ 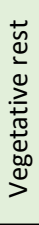 } & \multirow{2}{*}{ Jan } & c & \multicolumn{3}{|c|}{$60 \%$} & $67 \%$ & $82 \%$ & $96 \%$ & $110 \%$ & $124 \%$ & $139 \%$ & $153 \%$ & - \\
\hline & & $r$ & \multicolumn{3}{|c|}{-} & \multicolumn{7}{|c|}{$-*$} & $71 \%$ \\
\hline & \multirow{2}{*}{ Feb } & C & \multicolumn{3}{|c|}{$60 \%$} & $67 \%$ & $82 \%$ & $96 \%$ & $110 \%$ & $124 \%$ & $139 \%$ & $153 \%$ & - \\
\hline & & $r$ & \multicolumn{3}{|c|}{-} & \multicolumn{7}{|c|}{$-*$} & $71 \%$ \\
\hline & \multirow{2}{*}{ Mar } & C & \multicolumn{3}{|c|}{$60 \%$} & $67 \%$ & $82 \%$ & $96 \%$ & $110 \%$ & $124 \%$ & $139 \%$ & $153 \%$ & - \\
\hline & & $r$ & \multicolumn{3}{|c|}{-} & \multicolumn{7}{|c|}{$-*$} & $71 \%$ \\
\hline \multirow{4}{*}{$\begin{array}{l}3 \\
0 \\
\frac{3}{00} \\
\text { 00 } \\
. \frac{5}{2} \\
\text { ஸे }\end{array}$} & \multirow{2}{*}{ Apr } & C & \multicolumn{2}{|c|}{$45 \%$} & $71 \%$ & $93 \%$ & $107 \%$ & \multicolumn{6}{|c|}{-} \\
\hline & & $r$ & \multicolumn{2}{|c|}{-} & \multicolumn{3}{|c|}{$-*$} & \multicolumn{6}{|c|}{$148 \%$} \\
\hline & \multirow{2}{*}{ May } & c & \multicolumn{2}{|c|}{$45 \%$} & $71 \%$ & $93 \%$ & $107 \%$ & \multicolumn{6}{|c|}{-} \\
\hline & & $r$ & \multicolumn{2}{|c|}{-} & \multicolumn{3}{|c|}{$-*$} & \multicolumn{6}{|c|}{$148 \%$} \\
\hline \multirow{6}{*}{ 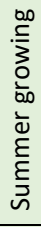 } & leun & C & $4 \%$ & $24 \%$ & $34 \%$ & & & & & & & & \\
\hline & Juil & $r$ & - & & & & & & $8 \varepsilon$ & & & & \\
\hline & $|u|$ & C & $4 \%$ & $24 \%$ & $34 \%$ & & & & & & & & \\
\hline & Juा & $r$ & - & & & & & & $8 \varepsilon$ & & & & \\
\hline & Aug & C & $4 \%$ & $24 \%$ & $34 \%$ & & & & & & & & \\
\hline & Aug & $r$ & - & & & & & & 88 & & & & \\
\hline 3 & Sen & C & $4 \%$ & $15 \%$ & $23 \%$ & $31 \%$ & $36 \%$ & & & & & & \\
\hline$\frac{0}{60}$ & sep & $r$ & - & & & & & & & 8 & & & \\
\hline 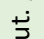 & Oct & C & $4 \%$ & $15 \%$ & $23 \%$ & $31 \%$ & $36 \%$ & & & & & & \\
\hline $\bar{\varangle}$ & Ut & $r$ & - & & & & & & & & & & \\
\hline$\ddot{\tilde{g}}$ & Nov & C & & $60 \%$ & & $67 \%$ & $82 \%$ & $96 \%$ & $110 \%$ & $124 \%$ & $139 \%$ & $153 \%$ & - \\
\hline 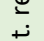 & NOV & $r$ & & - & & & & & $-*$ & & & & $71 \%$ \\
\hline ס్ & Der & C & & $60 \%$ & & $67 \%$ & $82 \%$ & $96 \%$ & $110 \%$ & $124 \%$ & $139 \%$ & $153 \%$ & - \\
\hline$>$ & Ded & $r$ & & - & & & & & $-*$ & & & & $71 \%$ \\
\hline
\end{tabular}

Figure S.15. Po Plain case: relative damage (Eq. 2 in the paper) to grassland for different combinations of times of flood occurrence (i.e. month), flood intensities (flood duration) and damage alleviation strategies ("c"=continuation; " $r "=$ reseeding). Results refer to a flood occurring before harvest has been made (under the hypothesis of 15 days remaining to mowing). 